\title{
LMB-7 Immunotoxin
}

National Cancer Institute

\section{Source}

National Cancer Institute. LMB-7 Immunotoxin. NCI Thesaurus. Code C1619.

A single chain chimeric protein consisting of a monoclonal antibody fragment attached to a portion of the Pseudomonas exotoxin A. LMB-7 immunotoxin attaches to B3, a Lewis Y-related carbohydrate epitope on some solid tumor cells. The antibody attaches to the cell and the exotoxin inhibits protein synthesis by inactivating elong ation factor 2. ( $\mathrm{NClO4)}$ 